\title{
Chemical Warehousing ERP System Design and Implementation
}

\author{
Guangmin Sun, Haitao Yan, Wei Liu, Rui Wang, Beichuan Ma, Junling Sun \\ Department of Electronic Engineering, Beijing University of Technology, Beijing, China \\ gmsun@bjut.edu.cn, haitaoyan@emails.bjut.edu.cn
}

\begin{abstract}
Chemical warehousing ERP system development is based on familiar chemical warehousing transit, storage, trade, business change, is effective tool to strengthen enterprise management and improve the work efficiency. This system is in Visual Studio2008 development environment, using c \# programming language, ASP.NET, ADO.NET and Oracle database to jointly develop a B/S structure of ERP system.

Index Terms - ASP.NET, ERP system, Oracle database, ADO.NET, B/S architecture

\section{Introduction}

ERP (Enterprise Resource Planning) is an integrative enterprise management software for material resources management (material flows), human resource management (people flows), financial resources management (financial flows), information resources management (information flows). ERP system can greatly improve the overall efficiency of the enterprise; maximize the sharing of resources, save a lot of human and financial resources. This paper designs and implements an chemical warehousing enterprise ERP system, through the enterprise resource management functional requirements analysis and design, hoping to improve enterprise management level of information and operating efficiency.
\end{abstract}

\section{The Overall System Analysis and Design}

A. System analysis

System overall analysis is roughly divided into the following three steps: (1) Research company sector institutions, including understand the composition of the company's departments, departments of responsibility etc., to prepare for the analysis of information flow. (2) Understand each department's business processes, including the understanding of the data needed for each sector, how to deal with these data, what is the output. (3) On the basis of the understanding of business activities, and interact with the company, clear requirement for ERP system, perfecting the functional requirements of each modules.

Based on detailed investigation of chemical warehousing enterprise business process, draw the chemical warehousing ERP system business flow chart and system function structure diagram, respectively figure 1 and figure 2, as shown below:

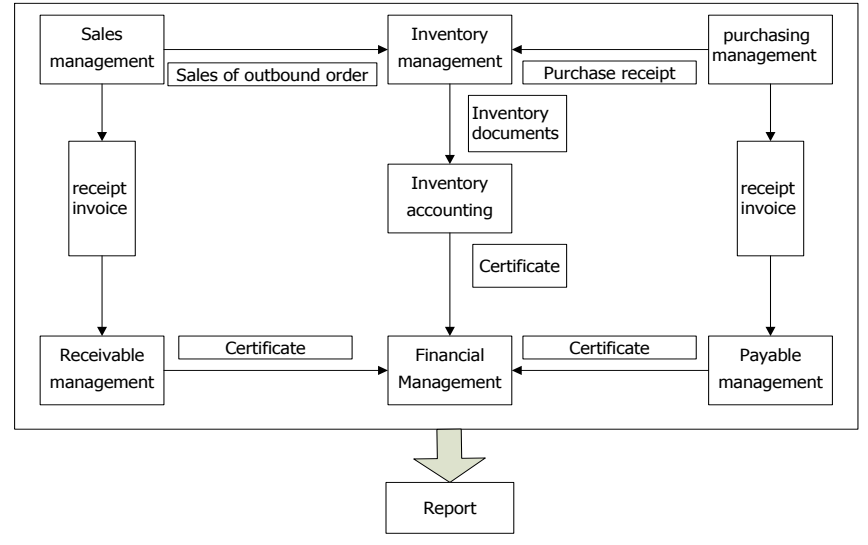

Fig.1 Chemical warehousing ERP system flow chart of the business

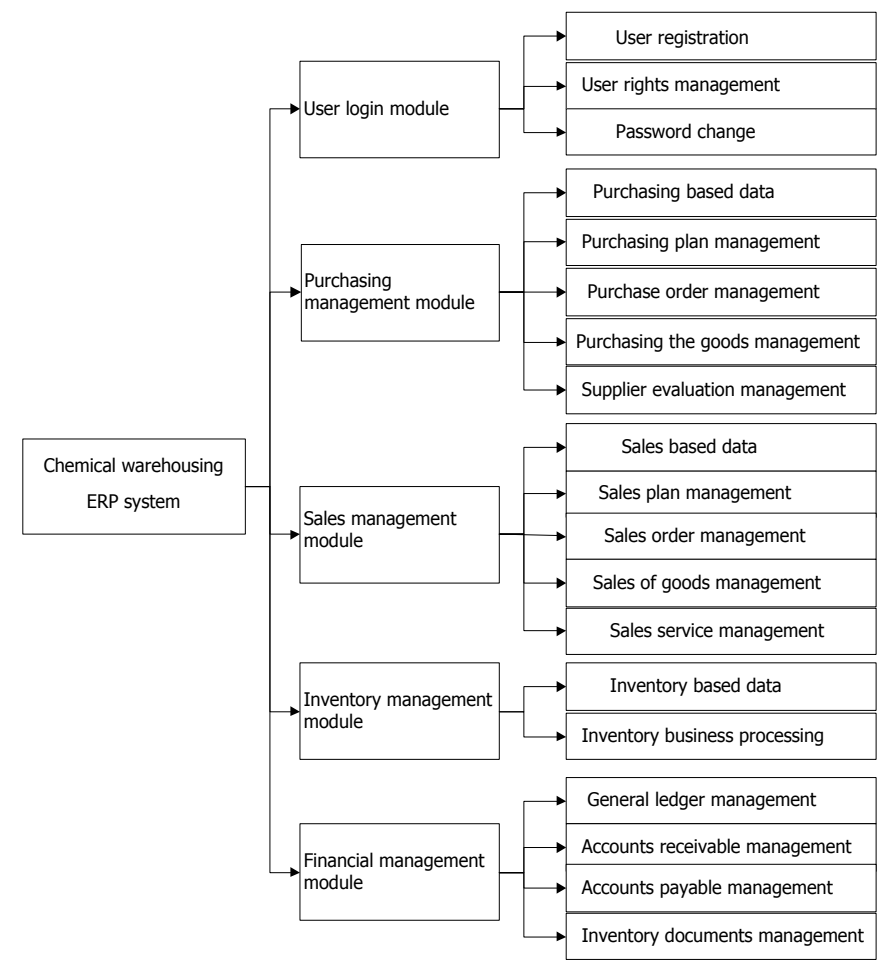

Fig.2 Chemical warehousing ERP system function structure diagram

\section{B. System development technical framework}

Chemical warehousing ERP system adopts B/S three layer architecture modes, with the rise of the internet technology, a change or improvement in the structure of $\mathrm{C} / \mathrm{S}$ architecture. Work under this architecture, the user interface is 
implemented via the WWW Browser, few transaction logic in the front-end (Browser), but the main transaction logic on the Server side (Server), forming the so-called three layer architecture, respectively for the presentation layer (UI), business logic layer (BLL), data access layer (DAL), as shown in figure 3 :

\begin{tabular}{c}
$\begin{array}{c}\text { Presentation } \\
\text { layer }\end{array}$ \\
\hline ASP. NET
\end{tabular}$\rightarrow$\begin{tabular}{c}
$\begin{array}{c}\text { Business logic } \\
\text { layer }\end{array}$ \\
\hdashline $\mathrm{C \#}$
\end{tabular}$\rightarrow$\begin{tabular}{c}
$\begin{array}{c}\text { Data access } \\
\text { layer } \\
\text { ADO. NET }\end{array}$ \\
Database \\
\hline
\end{tabular}

Fig.3 B/S three layer architecture

Presentation layer: Is located in the outermost layer (top), the most close to the user, is used to display data and receive user input data, to provide users with an interactive interface of the operation.

Business logic layer: A bridge between the UI layer and DAL layer plays an essential role in data exchange. Mainly for the operation of the specific question, the operation of the data layer, business logic for data processing, specific include: business logic validation, calculations, etc.

Data access layer: Its function is mainly responsible for database access, main realization of the data to add, delete, change, check, will be stored in the database data submitted to the business layer, at the same time, the business layer handles data saved to the database. (Of course, these operations are based on the UI layer. Reflect the needs of users to interface (UI), reflect the UI to the BLL, reflect the BLL to DAL, DAL for data operation, and after operation return one by one, until the user data needed feedback to the user).

\section{The Key Technical Analysis}

A. ASP.NET

ASP.NET is a unified Web application platform; it provides the necessary services for the establishment and deployment of enterprise Web application. ASP.NET provides a new programming model and infrastructure, can target any browser or device, more secure, more scalable, more stable.

ASP.NET is a part of Microsoft.NET Framework, is a compiled and based on the .NET Framework Environment, can use any with the .NET Framework compatible languages (including Visual Basic.NET, C \# and JScript.NET) authoring application. .NET Framework contains the common language runtime environment, type safety, inheritance, and so on, it provides a variety of core services, such as memory management, thread management and code security, it also contains the .NET Framework class library, this is an application developer to create a comprehensive, objectoriented collection of types, ASP.NET applications can use the entire .NET Framework, developers can easily get benefits of these technologies.

ASP.NET advantage is mainly manifested in the following aspects:

1) Managebility: ASP.NET uses a text-based, hierarchical configuration system, which simplifies applying settings to server environment and Web applications work.
Because the configuration information is stored as plain text, so you don't have a local management tools help also can apply the new settings. Any change in the configuration file can be automatically detected and used in the application.

2) Security: ASP.NET for Web applications provides a default authorization and authentication schemes. Developers can according to the needs of your application easily add, remove or replace these solutions.

\section{B. ADO.NET}

The ADO.NET name originated in ADO (ActiveX Data Objects), this is a broad class group, used to access the Data in the past the Microsoft technology. Why is named ADO.NET, because Microsoft wanted to show that this is in the .NET programming environment preferred data access interface. ADO.NET has the advantages of interoperability, maintainability, programmability, performance optimization, scalability, etc.

1) ADO.NET objects: Namely, the Connection object, Command object, DataAdapter object, DataSet object and DataReader object. These components responsible for creating online and data manipulation section we called data manipulation components (Managed Providers), respectively by the Connection object, Command object, DataAdapter object and DataReader object formed.

Data manipulation component the most main role is the bridge between the DataSet object and data source, responsible for the data in the data source after removing to implanted DataSet object, and store data back to the data source. Figure 4 is ADO.NET data object model diagram.

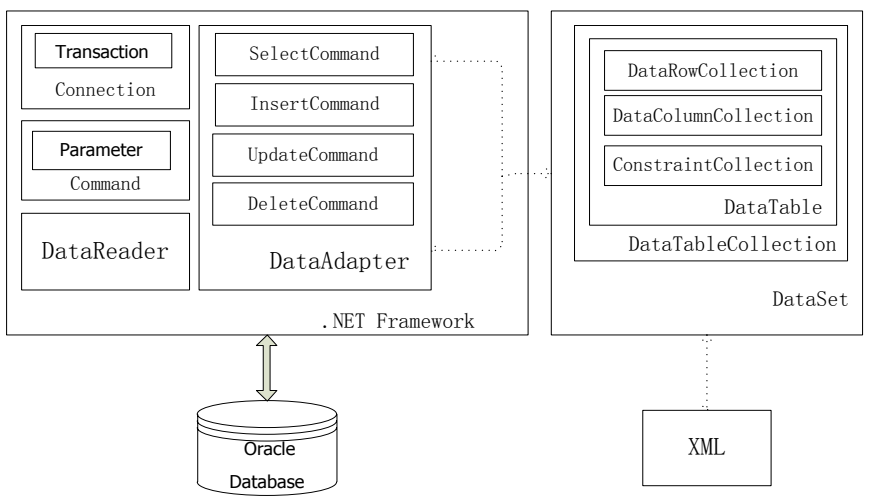

Fig.4 ADO.NET data object model diagram

As shown in figure 4 , the entire object model is divided into two parts. The first part is the .NET Framework data provider, the second part is the client local cache data sets.

First part: The .NET Framework data provider includes Connection object, Connection object can generate transaction object - Command object, and Command object associated with the parameter object, DataReader object, DataAdapter object, and DataAdapter object associated with the SelectCommand, InsertCommand, UpdateCommand, DeleteCommand object. Data provider is responsible for establishing connection, command, data transmission, etc. 
Second part: Local cache data sets consists of a DataSet object, the DataSet object in the local equivalent of a small database, so the DataSet object is composed of data table and table relationships, so the DataSet object contains the DataTable object and DataRelation object, and data table consists of rows, columns and constraints, so the DataTable object contains DataRow, DataColumn and Constraint object. Local cache data sets part can be used to temporarily store the local data, the data can be obtained from a Database also can be local produce, can also be modified data. In the case of using DataRelation and Constraint object, the client can access relational data such as access to the local cache data sets.

\section{Oracle DataBase}

Oracle database system is a distributed database as the core of a group of software products, is currently the most popular client/server or B/S structure of database system. Oracle Database represents data in the form of twodimensional table, and provides a SQL (structured query language), can complete basic database management functions, such as data query, operation, define and control.

Oracle Database features:

1) Oracle Database introduction of the Shared SQL and multithreading server architecture. This reduces the ORACLE resource usage, and enhances the ability of ORACLE, in cheap hardware and software platform with less resources can support more users, and on the high-end platform can support hundreds of thousands of users.

2) Provides the security management based on role division of labor. In the database management functions, integrity checking, security, consistency have good performance.

3) Support a large number of multimedia data, such as binary images, sound, animation, and multidimensional data structure, etc.

4) In $\mathrm{C} \#, \mathrm{C}++$ and other main language embedded SQL statements and procedural (PL / SQL) statement, to manipulate data in the database.

5) Provides a new distributed database capacity, can be more easily through the network to read and write the data in the remote database, and have symmetrical replication technology.

\section{The System Main Function}

\section{A. User login module}

User login module is to verify the identity of the user. Through which data is matched with the database, you can confirm the user's permission, according different permissions to different display interface, respectively into the corresponding interface, complete the corresponding interface initialization.

\section{B. Purchasing management module}

Procurement work mainly for the enterprise to provide the required materials, procurement management is the process of procurement business organization, implementation and control of the management process. Enterprise to provide the market with products or services is inseparable with material procurement, for enterprise, material costs accounted for a larger or very large proportion of the cost of the entire product, the purchasing department must find ways to reduce material procurement costs, must also consider the material inventory at the same time, a large number of inventory backlog items, means that the inventory cost of materials increase, capital takes up increase, so enterprises purchasing department through the feedback of sales and inventory, timely, right amount, proper price completion purchasing tasks, for the enterprise to provide needed materials. First, the purchasing department create purchasing plans, during the inquiry to negotiate, generate purchase payment plans, after checking by the financial department, approval issued a purchase orders, supplier according to the requirements of the orders to delivery of the goods, by the inventory manager to check and put in storage, finally out of the warehouse orders and purchase bills, and purchase bills forwarded to the finance department.

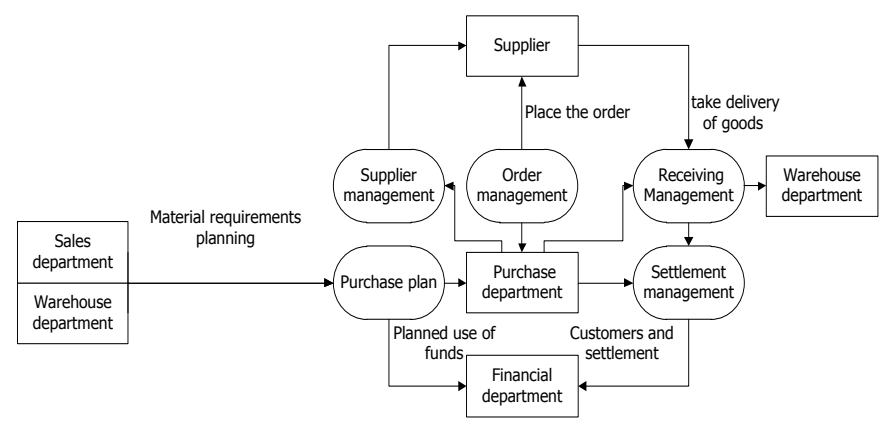

Fig.5 Procurement business process diagram

\section{Sales management module}

Marketing management main function is to provide products and services to customers and end users, make enterprises to obtain profits, provide enterprises with the power source of survival and development. Through formulating the plan of marketing and product quotation, according to the customer demand information, delivery information, product information and other considerations make sales orders, according to the sales orders in the order information, inventory personnel inventory allocation, organization for the customer to send the goods, finally out of the outbound order and sales bills, and sales bills forwarded to the finance department.

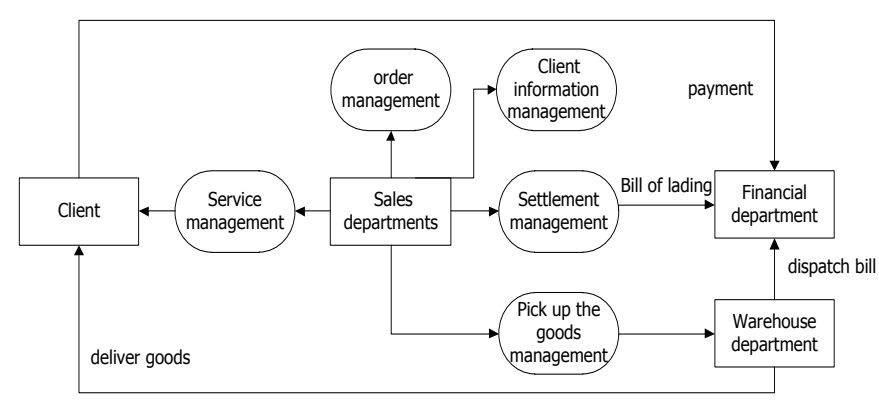

Fig.6 Sales business process diagram 


\section{Inventory management module}

Inventory management is the core of enterprise material management, is refers to the enterprise for the sake of procurement, sales need to plan the relevant goods storage and distribution management, such as the storage of items to receive, release, storage, safekeeping and a series of management activities. In sales and business operation activities of the enterprise, the enterprise inventory management mainly rises to balance the enterprise logistics, balance the circulation funds, and so on.

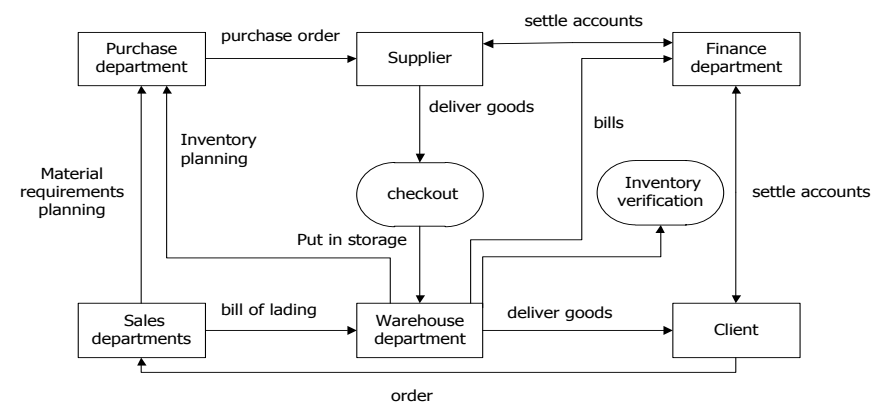

Fig.7 Inventory business process diagram

\section{E. Financial management module}

Enterprise financial management is based on the objective existence of the financial activities and financial relations, the financial management in the ERP system is integrated with procurement management, sales management, inventory management and all related to the enterprise financial activities. Its main function is to record and save every economic business original vouchers, after review the error-free, prepare accounting vouchers, and according to the business activities involved in financial subjects to fill in the corresponding certificate entries, after a period of time the certificates are classified into books for inspection. Production certificate and management is an important content of financial work, vouchers shall include original vouchers and recording vouchers, recording vouchers including receipt voucher, proof of payment and the transfer certificate. Financial management should be regular checking, to ensure true and reliable accounting records.

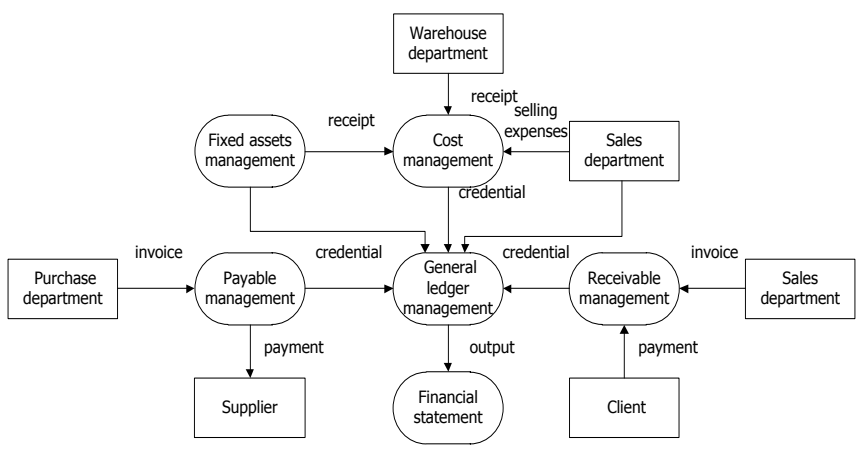

Fig.8 Financial business process diagram

\section{Conclusions}

In summary, the system uses the B / S three-tier technology development, security, stability and efficient. Can greatly reduce the work intensity of the enterprise employees, improve the efficiency of the enterprise staff, provides a reliable guarantee for the enterprise daily operation. At the same time, the employees can timely grasp of the company's operations, and can be done a series of audit and statistical work through the network, accurate, safe and efficient to create purchasing and sales plans, meet the various needs of users, and reflect the network information technology brings convenience operation.

\section{References}

[1] Ming Xue, Changjun Zhu, Shilong Song, The Development and Implementation of Household Textile Industry ERP System Based on B/S, IITA International Conference, 2009, Page(s): 655 - 658.

[2] Xuelian Yan, Changjun Zhu, Qing Yu Zhou, Research and Practice of Hydropower Engineering Construction Project ERP System Based on B/S, Pacific-Asia Conference, 2009, Page(s): 217 - 219.

[3] Wenjie Yang, Haoxue Liu, Jie Shi, The Design of Printing Enterprise Resources Planning (ERP) Software, The 2nd IEEE International Conference,2010, Page(s): $151-154$.

[4] Liwei Geng, Yujin Li, Yinan Wu, ERP-based Human Resource Management System, 2010 3rd International Conference on Advanced Computer Theory and Engineering(ICACTE), 2010, Page(s): V6-239 V6-241.

[5] JinMin Huang, The lab management system design and implementation based on ASP.NET, Computer CD Software and Applications, 2012.

[6] LiMing Zhang, Computer management information system course, Beijing University of technology press, 2006.

[7] LiMing Zhang, Computer software technology foundation, Beijing University of technology press, 2006. 